\title{
Effects of restraining measures due to COVID-19: Pre- and post-lockdown cognitive status and mental health
}

\author{
Joana Nogueira ${ }^{1,2} \cdot$ Bianca Gerardo ${ }^{1,2} \cdot$ Ana Rita Silva $^{1,2} \cdot$ Paula Pinto $^{2} \cdot$ Rosa Barbosa $^{1} \cdot$ Sílvia Soares $^{2}$. \\ Beatriz Baptista $^{3}$ - Carolina Paquete ${ }^{4}$ - Marina Cabral-Pinto ${ }^{5}$ Maria M. Vilar ${ }^{1,2}$ - Mário R. Simões ${ }^{1,2}$. \\ Sandra Freitas ${ }^{1,2}$ (iD
}

Accepted: 13 April 2021 / Published online: 21 April 2021

(C) The Author(s), under exclusive licence to Springer Science+Business Media, LLC, part of Springer Nature 2021

\begin{abstract}
The COVID-19 pandemic has prompted all countries to adopt restraining measures to mitigate the spread of the disease. Usually, large-scale disasters tend to be accompanied by significant increases of psychological distress, depression and anxiety. Confinement measures imposed during the COVID-19 pandemic are likely to have similar consequences. In the present study we aim to evaluate how COVID-19 affected the overall psychological functioning of Portuguese individuals by providing a comparison of current data with status prior to the COVID-19 pandemic. The study sample was composed of 150 cognitively healthy participants. Results show an overall maintenance of cognitive capacities, although subjective cognitive decline complaints significantly increased during the pandemic. Regarding mental health, restraining measures culminated in an aggravation of depressive and decrease of the perceived quality of life, associated with feelings of loneliness and perceived social isolation. Finally, higher levels of pre-COVID-19 quality of life seem to play a protective role against depression and anxiety and predict less difficulties in emotion regulation, feelings of solitude and cognitive complaints. In sum, confinement due to COVID-19 implied an aggravation of the mental health of the Portuguese population, which appears to have been attenuated in those with higher pre-pandemic levels of perceived quality of life.
\end{abstract}

Keywords COVID-19 · Pandemic · Social restrictions · Mental health · Cognition · Anxiety · Depression · Quality of life · Loneliness $\cdot$ Aging

Joana Nogueira and Bianca Gerardo contributed equally to this work.

\section{Sandra Freitas}

sandrafreitas0209@gmail.com

1 Center for Research in Neuropsychology and Cognitive and Behavioral Intervention (CINEICC), Faculty of Psychology and Educational Sciences (FPCEUC), Univ Coimbra, 3000-115 Coimbra, Portugal

2 Psychological Assessment and Psychometrics Laboratory (PsyAssessmentLab), Faculty of Psychology and Educational Sciences (FPCEUC), Univ Coimbra, 3000-115 Coimbra, Portugal

3 Centro de Terapia Familiar e Intervenção Sistémica de Ponta Delgada, São Miguel, Açores, Portugal

4 Faculty of Psychology and Educational Sciences (FPCEUC), Univ Coimbra, 3000-115 Coimbra, Portugal

5 Geobiotec Research Centre, Department of Geosciences, University of Aveiro, 3810-193 Aveiro, Portugal

\section{Introduction}

December 2019 was characterized by the uncovering of a novel virus that threatened human life. The severe acute respiratory syndrome coronavirus 2 (SARS-CoV-2) emerged in the city of Wuhan, China, and rapidly spread across the globe causing an outbreak of the coronavirus disease (COVID-19). Classified as a pandemic by the World Health Organization, COVID-19 reached all continents, having infected nearly 45 million individuals and caused 1 million plus deaths by October 2020. Ultimately, COVID-19 affected not only the health of individuals, but it jeopardized national health systems, economies, and societies.

By mid-March 2020, the anticipation of 6061 cases of COVID-19 led the Portuguese government to declare the state of emergency (Assembleia da República, 2020; DGS, 2020b). Similarly to other countries, Portugal went into lockdown, with several strategies being implemented in order to attempt the mitigation of the SARS-CoV-2 transmission. These strategies encompassed not only suitable responses of the national 
health care services, but most importantly the implementation of Public Health measures, such as restriction of movements, social distancing, social isolation, and quarantine (DGS, 2020a). Measures as such translated into extreme changes in lifestyle and relationship dynamics (e.g. detachment from loved ones, deprivation of individual liberties and routine disruption), imposing a heavy burden on the Portuguese people.

Regardless of their nature, large-scale disasters tend to be accompanied by significant increases of psychological distress, depression, anxiety, substance use, post-traumatic stress disorder (PTSD), violence, abuse and other mental and behavioral disorders (Cohen et al., 2017; Hawryluck et al., 2004; Jeong et al., 2016; Mak et al., 2010; Neria et al., 2008). Such issues typically occur immediately after the fact but may persist during long periods of time (Brooks et al., 2020; Galea et al., 2020). Confinement measures imposed during the COVID-19 pandemic are likely to have similar consequences. The precipitation of depression and anxiety associated with frustration, boredom, fear, uncertainty, and confusion is foreseen (Brooks et al., 2020), and individuals with pre-existing mental illnesses may be particularly affected (Venkatesh \& Edirappuli, 2020).

Recent research on COVID-19 already started to confirm such assumptions. For instance, studies conducted in China report that both the medical impact of the SARS-CoV-2 infection and the restraining measures play a role in anxiety levels, stress, depression, sleep-quality, PTSD and general well-being of its population (Liu et al., 2020; Xiao et al., 2020). A similar Italian study on the psychological cost of COVID-19 (Casagrande et al., 2020) pointed an increased risk for higher levels of anxiety symptomatology, psychological distress and sleep disturbances among younger individuals, women, individuals uncertain of having contracted the infection and people with a greater fear of contacting with those infected. Furthermore, sleep quality, generalized anxiety and psychological distress were found to be significantly associated with COVID-19-related PTSD symptomology (Casagrande et al., 2020). In Spain, González-Sanguino et al. (2020), found similar results, reporting the female gender, previous mental health issues/neurological disorders, symptoms compatible with COVID-19 and having a close relative infected by the coronavirus as positively associated with depression, anxiety and PTSD. Loneliness was highlighted as the strongest predictor of these symptoms, while older ages, economic stability, and trust in the quality of the information provided regarding COVID-19 were associated with less symptomatology (González-Sanguino et al., 2020). Interestingly, Huang and Zhao (2020) detected no gender differences in anxiety, depressive symptoms, and quality of sleep in the Chinese population, although their results corroborate literature findings of higher levels of generalized anxiety and depression among younger individuals. Notwithstanding, it is worthwhile noting that a thorough assessment of the effects of restraining measures due to COVID-19 requires a comparison with the populations' previous status (i.e., pre-pandemic). Studies assuming such design are lacking in the Portuguese population.

Whereas many studies have focused on this impact in emotional status and overall well-being of populations due to the restraints caused by COVID-19, other functional areas, such as objective cognitive functioning, are missing in current literature. To our knowledge, the question of whether the strategies implemented to counteract COVID-19 induced/will induce collateral damages in terms of the cognitive populations' abilities is not fully clarified. Previous research already highlighted how social isolation (Fratiglioni et al., 2004; Fratiglioni et al., 2000; Wang et al., 2002) and loneliness (Cacioppo \& Hawkley, 2009; O'luanaigh et al., 2012; Tilvis et al., 2004; Wilson et al., 2007) can be detrimental to cognition, accelerate cognitive decline and increase the risk for dementia, but less is known when this social isolation is mandatory due to the current matters. Furthermore, cognitive difficulties are common symptoms of psychological conditions and psychiatric disorders (Millan et al., 2012), so that previously described emotional disturbances due to COVID-19 counteracting strategies highlight the importance to deepen the understanding regarding the collateral effects of this reality in cognitive function and cognitive performance.

Since research studies on COVID-19 are lacking in the Portuguese population, we aim to evaluate how the pandemic disrupted individuals' mental health and general well-being and how it affected the cognitive status of adults and older adults. In that sense, a national cohort (already evaluated in 2018/2019) was reassessed during the term of restraining measures. This reassessment allows for the direct comparison of pre-pandemic and current data, enabling the study of the influence of COVID-19 pandemic on overall neuropsychological functioning of Portuguese population.

\section{Methodology}

\section{Participants and Procedures}

The total sample included 150 cognitively healthy participants (from a national cohort of 252) living in the Portuguese continental and island territory. All participants were invited to participate in the present study, having voluntarily accepted and given their informed consent. Since these participants were involved in previous studies that required the completion of a comprehensive cognitive assessment protocol, data on cognitive performance and psychopathology prior to the COVID-19 pandemic were available.

The inclusion criteria were the following: (i) being 50 years old or older; (ii) having at least one year of formal education; (iii) being a Portuguese native speaker, and (iv) absence of a 
psychiatric, neurological, or psychological clinical condition. The exclusion criteria included: (a) clinical conditions that affect cognitive performance (e.g.: neurological diseases, stroke, etc.); (b) intake of medication with an impact on cognitive functioning, and (c) presence of significant functional incapacity. There were 102 dropouts not included in the present study for several reasons, namely not being reachable by phone (34 participants), not wanting to be involved in the study (37 participants), having a clinical condition that hindered the participation (10 participants), and other motives (e.g., lack of computer resources, absence of support to carry out the evaluation, difficulties to deliver the questionnaires, etc.; 21 participants). For the present study, we did not exclude any clinical conditions due to COVID-19, as we only have a single positive asymptomatic case to report.

All 150 participants included in the final sample were reassessed by an experienced neuropsychologist. The time interval between the assessment moments (last cohort assessment prior to COVID-19 pandemic and the reassessed during the term of confinement measures) was approximately 1 year and 5 months $(514,89 \pm 75,53$ days).

Considering the social contacts' restrictions, the last assessment was implemented via video call $(n=111)$ or phone call $(n=39)$. The neuropsychological protocol was carefully adapted in order to minimize possible bias due to different assessment methods - since the previous evaluations were carried out in person, while this was not possible for the present study. As so, the participants who underwent a video call fulfilled all the cognitive tests that were included in the protocol, while the participants who were assessed by phone call only fulfilled the tests feasible to be applied by this method (i.e., the tasks that needed paper and pencil were excluded). The total score of the Mini-Mental State Examination (MMSE; Guerreiro et al., 1994) and the Montreal Cognitive Assessment (MoCA; Bosco et al., 2020; Folstein et al., 1975; Simões et al., 2008) applied via phone call were adapted regarding the number of tasks completed, i.e. their total scores excluded the punctuation of the paper-pencil tasks.

All participants signed an Informed Consent form that included a thorough explanation of the aims, procedures, and the confidentiality of the information provided during participation. The present research complied with the ethical guidelines for human experimentation stated in the Declaration of Helsinki and was approved by the Ethics Board and Scientific Committee of the affiliated Portuguese institutions.

\section{Measures}

All participants underwent a battery of instruments for the assessment of cognitive performance, functionality, presence of psychopathology, well-being and quality of life, and mental health literacy in social isolation.
A brief cognitive screening considering the MMSE and the MoCA was conducted in order to assess cognitive performance, and the following tools were administered for further exploration of cognitive abilities: the Trail Making Test (TMT-A/B; Cavaco et al., 2013), the Digit Symbol Coding (WAIS-III; Wechsler, 2008), the DigitSpan (WAIS-III; Wechsler, 2008), and the Fluencies Protocol (phonemic, semantic, letters inhibiting, and categories switching fluencies).

In addition, the participants fulfilled several questionnaires that allowed the examination of: (i) the functional capacity in basic and instrumental activities of daily living (IAFAI; Sousa et al., 2013); (ii) the presence of psychopathology, regarding depressive and anxiety symptomatology (Geriatric Depression Scale [GDS-30; Barreto et al., 2008] and Geriatric Anxiety Inventory [GAI; Ribeiro et al., 2011); (iii) the emotion regulation and vulnerability to stress (Difficulties in Emotion Regulation Scale [DERS; Coutinho et al., 2010] and Questionnaire of Vulnerability to Stress [23QVS; Vaz-Serra, 2000]); (iv) the quality of life (WHOQOL-OLD-7; Vilar et al., 2018). The assessment protocol also included a questionnaire of cognitive complaints (Cognitive Decline Complaints Scale, CDCS; Freitas et al., 2018). To further understand the effect of social restrictions, we included two questionnaires about social network and loneliness (respectively the Lubben Social Network Scale [LSNS; Ribeiro et al., 2012] and UCLA-Loneliness [Pocinho et al., 2010]). Finally, in order to better understand the knowledge of the Portuguese population regarding the pandemic crisis and its consequences (literacy), three new questionnaires were developed: (i) the Questionnaire of Literacy for Mental Health and Social Isolation, (ii) the Questionnaire of Literacy for COVID19, and (iii) the Questionnaire of Perceived Social Isolation (in which higher scores translate lower perceived social isolation).

\section{Statistical Analysis}

The statistical analysis was carried out using the Statistical Package for the Social Sciences (SPSS) for Windows, version 22 (IBM Corp., Armonk, N.Y., USA, 2013). Characterization of the sample was conducted through descriptive statistics (sociodemographic data, cognitive and non-cognitive profiles, new questionnaires data). Differences between the two moments of assessment were assessed through paired sample t-tests. A probability of $\leq$ .05 was assumed as significant in testing the null hypotheses of no differences between baseline and follow-up. Correlation analyses based on Pearson's correlation coefficients were performed to explore potential relationships between non-cognitive measures of the 2 nd assessment. Finally, effects of the pre-pandemic quality of life in non- 
cognitive consequences of lockdown were investigated through multiple linear regression (MLR) analyses (Enter method). A significance level of $95 \%$ was also used as criteria in both correlation and regression analyses.

\section{Results}

\section{Sample Characterization}

\section{Sociodemographic Data}

The mean age of the total sample was 69 years old $(69.02 \pm$ 7.95 ; [51-91]), $74.7 \%$ of females, with an average of approximately 9 years of formal education $(8.97 \pm 4.55$; [1-17]). In terms of residency, $80.2 \%$ of the sample lived in urban areas, while the remaining participants resided in rural areas (18.8\%).

Distinguishing between participants assessed via videoconference and via phone call, the former group presented a mean age of 68 years $(67.94 \pm 7.18$; [51-84]), and an average educational level of 10 years $(9.74 \pm 4.40$; [4-17]), with $93.9 \%$ living in urban areas. In turn, participants assessed via phone call were, on average, 72 years old $(72.10 \pm 9.23$; [54-91]) and had 7 years of formal education $(6.79 \pm 4.28$; [1-17]), with $81.0 \%$ living in urban areas.

\section{Cognitive Profile}

Cognitive characterization of the study sample is presented in Table 1. Considering the two methods of assessment (video call and phone call), values for the total sample were only presented for the cognitive tests that spared adaptation.

\section{Non-cognitive Profile}

Regarding the measures implemented to assess the presence of psychopathological symptoms, as well as other noncognitive variables, the total sample presented mild symptoms of depression (GDS-30: $8.13 \pm 6.26$ ) and anxiety (GAI: 7.63 $\pm 6.57)$. For quality of life, the sample showed a mean score of 28 points (WHOQOL-OLD-7: $28.16 \pm 3.79$ [control group: $29.47 \pm 4.32])$. The sample did not show relevant difficulties in emotion regulation (DERS: $38.01 \pm 21.25$ [normative data for 16-68 years old: $73 \pm 16]$ ), nor significant stress vulnerability (23QVS: $38.46 \pm 10.37$ [stress vulnerability $\geq 43$ ]). Finally, for the cognitive decline complaints scale, the sample showed a mean score of 24 points (CDCS: $24.49 \pm 15.16$ [control group: $14.86 \pm 10.48]$ ).

\section{New Questionnaires Data - COVID-19, Social Isolation and Health Literacy}

With a possible total score of 195 points, the average score obtained in the Questionnaire of Literacy for Mental Health and Social Isolation was of 145, approximately (144.88 \pm 11.89 [116-181]). With regard to the Questionnaire of Literacy for COVID-19, participants showed a mean score of 96 points $(95.93 \pm 5.95$ [84-112]) in a possible total of 135 points. Finally, participants exhibited, on average, a total score of 38 points for a maximum of 50 in the Questionnaire of Perceived Social Isolation (37.62 \pm 4.67 [22-48]).

\section{Comparisons between the 1st (before COVID-19) and 2nd Assessment (during COVID-19 Restraining Measures)}

\section{Cognitive Differences}

To fully understand the impact of experiencing confinement due to the COVID-19 pandemic, we performed a comparison between results obtained in two distinct moments of assessment: (i) the baseline, prior to the pandemic, and (ii) the follow-up, during COVID-19 restraining measures. The comparative analyses are detailed in Table 2. Significant differences were found regarding the MoCA total score of participants assessed through video call, with an increase in performance in the second assessment $(p=.020)$. Additionally, performances of the TMT significantly differed between the two evaluations, with participants taking longer to finish the tasks in the 2 nd assessment (TMT-A: $p=.002$; TMT-B: $p=.000$ ). The same pattern was found for the DSC (another measure of speed processing), for which we verified a significant decrease of the mean standardized score $(p=.002)$. It is important to note that these tasks were applied by video call in the second moment of assessment (to 111 participants). No other significant differences were found in the remaining tasks.

\section{Non-cognitive Differences}

Considering the enforcement of restraining social measures, we aimed to explore potential differences in subjective cognitive decline complaints, depressive symptomatology, and quality of life. The subjective memory decline complaints, assessed by the CDCS, suffered a significant increase from the $1 \mathrm{st}(21.75 \pm 14.44)$ to the $2 \mathrm{nd}$ assessment moment (24.49 $\left.\pm 15.16 ; t_{(82)}=-2.32, p=.023\right)$. The participants also exhibited a significant increase of depressive symptomatology in the 2nd assessment, according to the GDS-30 (1st assessment: $6.82 \pm 5.10 ; 2$ nd assessment: $8.13 \pm 6.26 ; t_{(141)}=-3.37$, $p=.001$ ), and a decrease in their perceived quality of life, according to the WHOQOL-OLD-7 (1st assessment: 29.64 
Table 1 Cognitive characterization of the sample during COVID-19 pandemic

\begin{tabular}{|c|c|c|c|}
\hline & $\begin{array}{l}\text { Video call } \\
(n=111)\end{array}$ & $\begin{array}{l}\text { Phone call } \\
(n=39)\end{array}$ & $\begin{array}{l}\text { Total Sample } \\
(n=150)\end{array}$ \\
\hline MMSE & $28.88 \pm 1.41$ & $20.59 \pm 2.13$ & - \\
\hline \multirow[t]{2}{*}{$\mathrm{MoCA}$} & $\begin{array}{l}{[23-30]} \\
24.26 \pm 3.78\end{array}$ & $\begin{array}{l}{[13-23]} \\
16.08 \pm 3.30\end{array}$ & - \\
\hline & {$[9-30]$} & {$[9-22]$} & \\
\hline \multirow[t]{2}{*}{ MIS - MoCA } & $11.59 \pm 3.29$ & $11.46 \pm 3.73$ & $11.56 \pm 3.40$ \\
\hline & {$[2-15]$} & {$[2-15]$} & {$[2-15]$} \\
\hline \multirow[t]{2}{*}{ CDT } & $13.47 \pm 4.24$ & - & - \\
\hline & {$[2-18]$} & & \\
\hline \multirow[t]{2}{*}{ TMT-A } & $58.10 \pm 28.03$ & - & - \\
\hline & {$[0-137]$} & & \\
\hline \multirow[t]{2}{*}{ TMT-B } & $141.01 \pm 81.40$ & - & - \\
\hline & {$[0-380]$} & & \\
\hline \multirow[t]{2}{*}{ DSC } & $11.93 \pm 2.68$ & - & - \\
\hline & {$[5-19]$} & & \\
\hline \multirow[t]{2}{*}{ Digit-Span forward } & $8.29 \pm 2.49$ & $8.09 \pm 2.06$ & $8.24 \pm 2.39$ \\
\hline & {$[5-16]$} & {$[5-14]$} & {$[5-16]$} \\
\hline \multirow[t]{2}{*}{ Digit-Span backwards } & $5.69 \pm 2.72$ & $5.43 \pm 2.96$ & $5.63 \pm 2.77$ \\
\hline & {$[1-14]$} & {$[1-14]$} & {$[1-14]$} \\
\hline \multirow[t]{2}{*}{ Digit-Span total } & $12.14 \pm 3.31$ & $12.31 \pm 3.23$ & $12.18 \pm 3.28$ \\
\hline & {$[6-19]$} & [7-19] & {$[6-19]$} \\
\hline \multirow[t]{2}{*}{ Letter Fluency - P } & $11.29 \pm 4.65$ & $9.49 \pm 5.27$ & $10.86 \pm 4.85$ \\
\hline & {$[2-25]$} & {$[2-23]$} & {$[2-25]$} \\
\hline \multirow[t]{2}{*}{ Letter Fluency - M } & $10.25 \pm 4.43$ & $9.53 \pm 4.80$ & $10.08 \pm 4.52$ \\
\hline & {$[3-22]$} & {$[2-22]$} & {$[2-22]$} \\
\hline \multirow[t]{2}{*}{ Letter Fluency - R } & $9.70 \pm 4.99$ & $9.26 \pm 4.57$ & $9.60 \pm 4.88$ \\
\hline & [1-23] & {$[1-17]$} & {$[1-23]$} \\
\hline \multirow[t]{2}{*}{ Category Fluency - Animals } & $17.84 \pm 5.68$ & $15.17 \pm 5.74$ & $17.18 \pm 5.79$ \\
\hline & {$[1-31]$} & {$[4-30]$} & {$[1-31]$} \\
\hline \multirow[t]{2}{*}{ Category Fluency - Food } & $19.02 \pm 5.86$ & $17.88 \pm 6.44$ & $18.74 \pm 6.00$ \\
\hline & {$[0-38]$} & {$[5-30]$} & {$[0-38]$} \\
\hline \multirow[t]{2}{*}{ Category Fluency - Actions } & $13.34 \pm 6.18$ & $10.23 \pm 5.36$ & $12.57 \pm 6.12$ \\
\hline & {$[2-30]$} & {$[2-28]$} & {$[2-30]$} \\
\hline \multirow[t]{2}{*}{ Letters Inhibiting Fluency - A } & $6.07 \pm 3.61$ & $4.94 \pm 2.90$ & $5.79 \pm 3.47$ \\
\hline & {$[0-16]$} & {$[0-13]$} & {$[0-16]$} \\
\hline \multirow[t]{2}{*}{ Letter Inhibiting Fluency - E } & $9.40 \pm 3.72$ & $8.00 \pm 3.77$ & $9.06 \pm 3.76$ \\
\hline & {$[2-18]$} & {$[0-15]$} & {$[0-18]$} \\
\hline \multirow[t]{2}{*}{ Letter Inhibiting Fluency - I } & $11.77 \pm 5.06$ & $9.88 \pm 4.17$ & $11.32 \pm 4.92$ \\
\hline & {$[1-25]$} & {$[1-18]$} & {$[1-25]$} \\
\hline Categories Switching & $12.47 \pm 3.29$ & $10.52 \pm 3.58$ & $12.01 \pm 3.45$ \\
\hline Furniture - Fruits & {$[6-19]$} & {$[3-16]$} & {$[3-19]$} \\
\hline \multirow[t]{2}{*}{ Categories Switching Professions - Colours } & $14.38 \pm 3.94$ & $10.91 \pm 4.54$ & $13.55 \pm 4.34$ \\
\hline & {$[0-31]$} & {$[3-20]$} & {$[0-31]$} \\
\hline
\end{tabular}

MMSE (Mini-Mental State Examination); MoCA (Montreal Cognitive Test); MIS-MoCA (memory index score); CDT (Clock-drawing test); TMT-A/B (Trail Making Test-A/B expressed in time of performance by seconds), DSC (Digit Symbol Coding expressed in standardized scores), Digit-Span (expressed in standardized scores), Fluencies (total of words in $1 \mathrm{~min}$ ) $\pm 3.83 ; 2$ nd assessment: $\left.28.12 \pm 3.77 ; t_{(123)}=4.78, p<.001\right)$. Furthermore, we explored the number of social contacts before and during the quarantine using the LSNS. Considering the differential acquired in the LSNS, $83.2 \%$ of participants increased or maintained their social contacts, while $16.8 \%$ decreased their social contacts during the lockdown. 
Table 2 Cognitive differences between the 1 st and the 2 nd assessment

\begin{tabular}{|c|c|c|c|c|}
\hline & & 1 st assessment & 2 nd assessment & Group differences \\
\hline \multirow{6}{*}{$\begin{array}{l}\text { Video call } \\
(n=111)\end{array}$} & MMSE & $28.67 \pm 1.45$ & $28.87 \pm 1.42$ & $t_{(104)}=-1.34, p=.183$ \\
\hline & $\mathrm{MoCA}$ & $23.24 \pm 3.74$ & $24.08 \pm 3.83$ & $t_{(85)}=-2.37, p=.020$ \\
\hline & CDT & $13.71 \pm 4.04$ & $13.63 \pm 4.077$ & $t_{(93)}=.184, p=.854$ \\
\hline & TMT-A & $49.09 \pm 18.83$ & $60.10 \pm 29.31$ & $t_{(72)}=-3.23, p=.002$ \\
\hline & TMT-B & $109.30 \pm 48.84$ & $144.79 \pm 81.71$ & $t_{(65)}=-4.53, p=.000$ \\
\hline & DSC & $12.49 \pm 2.48$ & $11.67 \pm 2.56$ & $t_{(66)}=3.17, p=.002$ \\
\hline \multirow{14}{*}{$\begin{array}{l}\text { Total Sample } \\
(n=150)\end{array}$} & MMSE (adapted) & $20.98 \pm 1.25$ & $21.08 \pm 1.28$ & $t_{(131)}=-.91, p=.364$ \\
\hline & MoCA (adapted) & $16.51 \pm 3.14$ & $16.92 \pm 2.99$ & $t_{(131)}=-1.69, p=.093$ \\
\hline & DSC & $12.14 \pm 3.31$ & $12.31 \pm 3.23$ & $t_{(87)}=1.72, p=.090$ \\
\hline & Letter Fluency - P & $11.29 \pm 4.65$ & $9.49 \pm 5.27$ & $t_{(127)}=1.33, p=.188$ \\
\hline & Letter Fluency - M & $10.25 \pm 4.43$ & $9.53 \pm 4.80$ & $t_{(76)}=1.69, p=.095$ \\
\hline & Letter Fluency - R & $9.70 \pm 4.99$ & $9.26 \pm 4.57$ & $t_{(77)}=.841, p=.403$ \\
\hline & Categories Fluency - Animals & $17.84 \pm 5.68$ & $15.17 \pm 5.74$ & $t_{(80)}=-.133, p=.894$ \\
\hline & Categories Fluency - Food & $19.02 \pm 5.86$ & $17.88 \pm 6.44$ & $t_{(77)}=-.761, p=.449$ \\
\hline & Categories Fluency - Actions & $13.34 \pm 6.18$ & $10.23 \pm 5.36$ & $t_{(78)}=-.366, p=.716$ \\
\hline & Letters Inibithing Fluency - A & $6.07 \pm 3.61$ & $4.94 \pm 2.90$ & $t_{(75)}=-1.801, p=.076$ \\
\hline & Letters Inhibiting Fluency - E & $9.40 \pm 3.72$ & $8.00 \pm 3.77$ & $t_{(76)}=-2.19, p=.031$ \\
\hline & Letters Inhibiting Fluency- I & $11.77 \pm 5.06$ & $9.88 \pm 4.17$ & $t_{(75)}=-.729, p=.468$ \\
\hline & Categories Switching Professions- Colours & $12.47 \pm 3.29$ & $10.52 \pm 3.58$ & $t_{(73)}=.467, p=.642$ \\
\hline & Categories Switching Furniture-Fruits & $14.38 \pm 3.94$ & $10.91 \pm 4.54$ & $t_{(72)}=1.87, p=.066$ \\
\hline
\end{tabular}

MMSE (Mini-Mental State Examination); MoCA (Montreal Cognitive Test); CDT (Clock-drawing test); TMT-A/B (Trail Making Test-A/B expressed in time of performance by seconds), DSC (Digit Symbol Coding expressed in standardized scores), Digit-Span (expressed in standardized scores), Fluencies (total of words in $1 \mathrm{~min}$ )

\section{Associations between Measures}

In order to assess whether non-cognitive measures correlated with each other, as well as with literacy indicators, correlation analyses were performed. In regard to the $2 \mathrm{nd}$ assessment, for the UCLA-16 total scores we found significant positive correlations with: (i) GDS-30 total scores $(r=0.536, p<.001)$; (ii) GAI total scores $(r=.350$, $p<.001)$; (iii) CDCS total scores $(r=.440, p<.001)$; (iv) QVS23 total scores $(r=.494, p<.001)$, and (v) DERS total scores $(r=.324, p<.001)$. Also, the UCLA-16 total scores were negatively correlated with the WHOQOL-OLD-7 total scores $(r=-.486, p<.001)$ and with the Questionnaire of Perceived Social Isolation $(r=-.342, p<.001)$. In turn, scores obtained on the Questionnaire of Perceived Social Isolation were positively correlated with the WHOQOLOLD-7 total scores $(r=.267, p=.002)$ and the Questionnaire of Literacy for Mental Health and Social Distancing $(r=.304, p<.001)$, while negatively correlated with the GDS-30 $(r=-.296, p<.001)$. The LSNS-during COVID-19 correlated positively with the Questionnaire of Perceived Social Isolation $(r=.332, p<.001)$ and negatively with the UCLA-16 $(r=-.494, p<.001)$.

\section{Effect of Pre-Pandemic Quality of Life in the Non- cognitive Consequences of Lockdown}

In order to assess whether the perceived quality of life prior to the COVID-19 pandemic significantly affected psychopathological symptomatology, emotional regulation, vulnerability to stress and loneliness, as well as cognitive complaints, during the enforcement of lockdown, linear regression analyses were performed.

Regarding psychopathological symptoms, namely depression and anxiety, the WHOQOL-OLD-7 (1st assessment) revealed to be a significant predictor of both the GDS-30 $(\beta=-.595, t=-8.12, p<.001)$ and the GAI $(\beta=-.2 .80, t=$ $-3.26, p=.001$ ) total scores (2nd assessment). While an unit decrease in the WHOQOL-OLD-7 corresponded to an increase of .83 points in the GDS-30 total score, 1 point less in the WHOQOL-OLD-7 represented a gain of .46 points in the GAI global score. Both models were statistically significant $\left(\right.$ GDS-30: $F_{(1,120)}=65.93, p<.001 ;$ GAI: $F_{(1,125)}=$ $10.61, p=.001$ ), with the WHOQOL-OLD-7 explaining approximately $35 \%\left(\mathrm{R}_{\text {adj }}^{2}=.349\right)$ and $7 \%\left(\mathrm{R}^{2}{ }_{\text {adj }}=.071\right)$ of the variances observed in the GDS-30 and GAI scores, respectively. 
Regressions models also showed the WHOQOL-OLD-7 (1st assessment) as a significant predictor of DERS scores during COVID-19 ( $(B=-.307, t=-3.51, p=.001)$, with a unit decrease in the former predicting an increase of 1.17 points in the latest (in a statistically significant linear model: $\left.\mathrm{R}^{2}{ }_{\text {adj }}=.087, F_{(1,118)}=12.31, p=.001\right)$. The same was true for the QVS23 $(B=-.344, t=-4.01, p<.001)$ and for the UCLA-16 ( $\beta=-.395, t=-4.73, p<.001)$ total scores, for which each 1 point decrease in WHOQOL-OLD-7 (1st assessment) translated an increase of .89 and .82 points in the total scores, respectively (in statistically significant linear models; QVS23: $\mathrm{R}_{\text {adj }}^{2}=.111, F_{(1,120)}=16.09, p<.001$; UCLA-16: $\left.\mathrm{R}_{\text {adj }}^{2}=.149, F_{(1,121)}=22.39, p<.001\right)$.

Finally, analyses revealed that the WHOQOL-OLD-7 total scores acquired before the pandemic significantly predict the global scores obtained in the CDCS $(\beta=-.395, t=-4.73$, $p<.001)$. In this case, 1 point less in the former instrument translated into a gain of 1.58 points in the total score of the latest. The regression model was statistically significant $\left(F_{(1,121)}=22.34, p=.001\right)$, with the WHOQOL-OLD-7 explaining $14.9 \%$ of the variance observed in the CDCS scores.

\section{Discussion}

The COVID-19 pandemic has prompted all countries to adopt restraining measures to attempt the mitigation of the SARSCoV-2 transmission, which culminated in various forms of populations' confinement. In Portugal, such measures implied one and a half months of full lockdown, as well as the enforcement of other restrictions (of various degrees) that are being enforced to this date. The present study aimed to evaluate how COVID-19 affected the overall psychological functioning of Portuguese individuals by providing a comparison of current data with the status prior to the pandemic, addressing cognitive functioning, mental health, and general well-being.

With regard to the effects of lockdown in cognitive functioning, our results suggest an overall maintenance of these capacities. The MoCA total score obtained from video call assessments was the only screening result to vary from baseline to follow-up, a difference that faded when the total sample was considered. Such observation may be partially imputed to the differences on the interface used for both assessments (as it was different from baseline [face-to-face] to follow-up), but not totally, as recent studies concluded for the reliability of video call relative to face-to-face assessments in similar populations (Castanho et al., 2016; Stead \& Vinson, 2019). In terms of specific cognitive measures, participants exhibited deterioration of cognitive flexibility and processing speed (indexed by longer completion times on the TMT [A and B] and fewer correctly repeated sequences on DSC), when only video call assessments were analysed. However, differences were not found for the generality of performances when the total sample was considered. Such results of cognitive maintenance are unexpected, considering that previous research highlights how social isolation and loneliness (Cacioppo \& Hawkley, 2009; Fratiglioni et al., 2000, 2004; O'luanaigh et al., 2012; Tilvis et al., 2004; Wang et al., 2002; Wilson et al., 2007) may accelerate cognitive decline. Interestingly, participants reported significantly more subjective cognitive decline complaints during COVID-19, despite the maintenance of their overall cognitive functioning. Correlation analyses revealed that this perception of cognitive decline is associated with experiencing feelings of loneliness (indexed by UCLA-16). In other words, our findings suggest that deeper feelings of loneliness are associated with exacerbated complaints that do not translate objective cognitive decline.

Concerning non-cognitive measures, participants seem to exhibit mild symptoms of depression and anxiety. Results on differences between the pre-pandemic (baseline) and the current period of COVID-19 (follow-up) evidence a significant increase of depressive symptomatology and decrease of perceived quality of life. While more symptoms of depression seem to be associated with deeper feelings of solitude, lower quality of life correlated with feelings of solitude and the perception of a greater social isolation. In turn, observations of the LSNS results reveal that the majority of the study sample maintained or increased the number of social interaction during lockdown and following restraining measures, which may account as a protective factor for the presence of only mild symptoms of anxiety and depression. Despite social restrictions, research supports the significant role of social networks, for instance maintained by means of technology, in the preservation of a sense of connectedness and overall well-being (Saltzman et al., 2020; Van Orden et al., 2020). White and Van Der Boor (2020) have previously described a decrease in the perceived well-being and quality of life of UK citizens during the initial weeks of lockdown. However, in their study, feelings of community connectedness assumed a protective role, contributing for better perceptions of well-being. In our study, significant correlations were indeed found between greater magnitudes of social networks and lower perception of social isolation and feelings of solitude.

Correlation analyses also showed that, doing the enforcement of restraining measures due to COVID-19, subjective feelings of loneliness are significantly associated with more severe symptoms of anxiety, greater vulnerability to stress, difficulties in emotion regulation, perception of greater social isolation and fewer social contacts. In turn, the perception of social isolation associate with not only fewer social interactions, but also with lower literacy for mental health and social distancing. Together, these findings are in line with previous evidence supporting the contribution of social isolation and restraining measures to the increase of anxiety and depressive symptomatology, as well as perceived loneliness on healthy 
adults and older adults (Liu et al., 2020; Tull et al., 2020; Xiao et al., 2020). A recent study on the Spanish population reported loneliness as the strongest predictor of depression, anxiety, and PTSD (González-Sanguino et al., 2020). Current correlation analyses highlight how feelings of loneliness and perceived social isolation not only relate to the number of social interactions, but also to the vulnerability to stress, difficulties in emotion regulation and literacy for mental health and social distancing.

Since by definition, quality of life is considered to be "the individual's perception of their position in life in the context of the culture and value systems in which they live and in relation to their goals, expectations, standards and concerns" (WHOQOL Group, 1995), an influence of such factor in the facing of the COVID-19 pandemic was foreseen. Regression analyses on the effects of pre-pandemic quality of life on the non-cognitive consequences of lockdown revealed poorer preCOVID quality of life as a relevant predictor of greater symptomatology of depression and anxiety. Additionally, poorer quality of life significantly predicted greater difficulties in emotion regulation, vulnerability to stress, feelings of loneliness, and subjective cognitive decline complaints. Quality of life is a key element when facing adversity. Resilience is defined by the American Psychological Association (2011) as "a successful adaptation process in response to threatening, stressful, or traumatic adverse experiences, or the ability to bounce back from difficult life conditions". As previous research has shown, high resilience later in life is associated with not only greater happiness, mental health and greater well-being, but also with increased quality of life (Bowling \& Iliffe, 2011; Hildon et al., 2010; Netuveli \& Blane, 2008; Nygren et al., 2005). A recent study suggests that quality of life may be affected by loneliness via two different ways, resilience and mental health (Gerino et al., 2017), which may explain the drop in participants' perceived quality of life that was observed during COVID-19 (as symptoms of depression also rose).

Some limitations to the present study were pointed out, namely: (i) the inclusion of only individuals with ages equal or above 50 years (although the study sample was representative for this age group, since it was stratified according to the sociodemographic variables), which led to the exclusion a younger adults, (ii) the usage of remote methods to apply neuropsychological assessments, online or otherwise, since these were not the same methods used at the baseline, and (iii) the inability to reassess the entirety of the sample by video call.

\section{Conclusions}

The current study provides original evidence on the effects of COVID-19 lockdown in the cognitive status and mental health of the Portuguese population through a longitudinal design that increases the reliability of results, given the availability of baseline characteristics of the study sample.

Overall, restraining measures culminated in the aggravation of depressive symptomatology and decrease of the perceived quality of life, associated with feelings of loneliness and perceived social isolation. Despite social networks not being greatly affected by the pandemic, such feelings of loneliness and perceptions of social isolation appear to be associated with anxiety, vulnerability to stress, difficulties in regulating emotions and literacy for mental health and social distancing. Additionally, subjective cognitive decline complaints significantly increased during the pandemic, regardless of the relative preservation of cognitive capacities. More favorable perceptions of pre-COVID-19 quality of life seem to play a protective role against depression and anxiety and predict less difficulties in emotion regulation, feelings of solitude and cognitive complaints, as well as greater resistance to stress during restraining measures.

Future studies on the psychological effects of COVID-19 should consider similar longitudinal designs, as the comparison with conditions prior to the pandemic are crucial to fully grasp its consequences. Further age groups should also be included to ensure greater generalization of results.

Funding This work was supported by the RESEARCH4COVID 19 (FCT/RESEARCH4COVID19/079_596650513) from Foundation for Science and Technology [S.F., grant number: IF/01325/2015], [J.N., grant number: SFRH/BD/136029/2018], [B.G., grant number: $\mathrm{SFRH} / \mathrm{BD} / 146680 / 2019]$.

Data Availability The datasets generated during and/or analysed during the current study are not publicly available due to the impossibility of a complete anonymization of participants' data, but are available from the corresponding author on reasonable request.

\section{Declaration}

Conflict of Interest The authors declare no conflict of interest.

\section{References}

American Psychological Association (APA). (2011). The road to resilience. Available online at: http://www.apa.org/helpcenter/roadresilience.aspx

Assembleia da República. (2020). Estado de emergência. Retrieved from https://www.parlamento.pt/Paginas/2020/marco/Estado-deemergencia.aspx $? \mathrm{n}=20$

Barreto, J., Leuschner, A., Santos, F., \& Sobral, M. (2008). Escala de Depressão Geriátrica. In Grupo de Estudos de Envelhecimento Cerebral e Demência (Ed.), Escalas e Testes na Demência (pp. 6972). Lisboa: Novartis.

Bosco, A., Caffò, A. O., Spano, G., Lopez, A. (2020). Beyond the cutoffs: A Bayesian approach to the use of MoCA as a screening tool for MCI and Dementia. V. R. Preedy \& C. R. Martin (Eds.), The 
Neuroscience of Dementia: Diagnosis and Management. Elsevier. ISBN 978-0-12-815854-8.

Bowling, A., \& Iliffe, S. (2011). Psychological approach to successful ageing predicts future quality of life in older adults. Health and Quality of Life Outcomes, 9(1), 13.

Brooks, S. K., Webster, R. K., Smith, L. E., Woodland, L., Wessely, S., Greenberg, N., \& Rubin, G. J. (2020). The psychological impact of quarantine and how to reduce it: Rapid review of the evidence. The Lancet, 395(10227), 14-20. https://doi.org/10.1016/S01406736(20)30460-8.

Cacioppo, J. T., \& Hawkley, L. C. (2009). Perceived social isolation and cognition. Trends in Cognitive Sciences, 13(10), 447-454. https:// doi.org/10.1016/j.tics.2009.06.005.

Casagrande, M., Favieri, F., Tambelli, R., \& Forte, G. (2020). The enemy who sealed the world: Effects quarantine due to the COVID-19 on sleep quality, anxiety, and psychological distress in the Italian population. Sleep Medicine., 75, 12-20. https://doi.org/10.1016/j.sleep. 2020.05.011.

Castanho, T. C., Amorim, L., Moreira, P. S., Mariz, J., Palha, J. A., Sousa, N., \& Santos, N. C. (2016). Assessing cognitive function in older adults using a videoconference approach. EBioMedicine, 11, 278-284. https://doi.org/10.1016/j.ebiom.2016.08.001.

Cavaco, S., Gonçalves, A., Pinto, C., Almeida, E., Gomes, F., Moreira, I., et al. (2013). Trail making test: Regression-based norms for the Portuguese population. Archives of Clinical Neuropsychology, 28, 189-198.

Cohen, S., Kaplan, Z., Zohar, J., \& Cohen, H. (2017). Preventing sleep on the first resting phase following a traumatic event attenuates anxietyrelated responses. Behavioural Brain Research, 320, 450-456.

Coutinho, J., Ribeiro, E., Ferreirinha, R., \& Dias, P. (2010). The Portuguese version of the difficulties in emotion regulation scale and its relationship with psychopathological symptoms. Archives of Clinical Psychiatry (São Paulo), 37(4), 145-151.

Direção-Geral da Saúde, DGS (2020a). Plano Nacional de Preparação e Resposta à Doença por novo coronavírus (COVID-19). Retrieved from: https://covid19.min-saude.pt/plano-de-contingencia/

Direção-Geral da Saúde, DGS (2020b). Relatório de Situação $n^{\circ} 017$. Retrieved from: https://covid19.min-saude.pt/relatorio-de-situacao/

Folstein, M., Folstein, S., \& McHugh, P. (1975). Mini-mental state: A practical method for grading the cognitive state of patients for the clinician. Journal of Psychiatry Research, 12(3), 189-198. https:// doi.org/10.1016/0022-3956(75)90026-6.

Fratiglioni, L., Paillard-Borg, S., \& Winblad, B. (2004). An active and socially integrated lifestyle in late life might protect against dementia. Lancet Neurology, 3, 343-353. https://doi.org/10.1016/S14744422(04)00767-7.

Fratiglioni, L., Wang, H.X., Ericsson, K., Maytan, M., \& Winblad, B. (2000). Influence of social network on occurrence of dementia. Lancet, 355, 1315-1319. https://doi.org/10.1016/S0140-6736(00) 02113-9.

Freitas, S., Nogueira, J., Prieto, G., Vilar, M., Simões, M. R., \& Santana, I. (2018). Cognitive Decline Complaints Scale (CDCS) / Escala de Queixas de Declínio Cognitivo (EQDC). PsyLabAssessment, Faculdade de Psicologia e de Ciências da Educação da Universidade de Coimbra. Portugal.

Galea, S., Merchant, R. M., \& Lurie, N. (2020). The mental health consequences of COVID-19 and physical distancing: The need for prevention and early intervention. JAMA Internal Medicine, 180(6), 817-818. https://doi.org/10.1001/jamainternmed.2020.1562.

Gerino, E., Rollè, L., Sechi, C., \& Brustia, P. (2017). Loneliness, resilience, mental health, and quality of life in old age: A structural equation model. Frontiers in Psychology, 8, 2003. https://doi.org/ 10.3389/fpsyg.2017.02003.

González-Sanguino, C., Ausín, B., ÁngelCastellanos, M., Saiz, J., LópezGómez, A., Ugidos, C., \& Muñoz, M. (2020). Mental health consequences during the initial stage of the 2020 coronavirus pandemic
(COVID-19) in Spain. Brain, Behavior, and Immunity, 87, 172176. https://doi.org/10.1016/j.bbi.2020.05.040.

Guerreiro, M., Silva, A. P., Botelho, M. A., Leitão, O., Castro-Caldas, A., \& Garcia, C. (1994). Adaptação à população portuguesa da tradução do "Mini Mental State Examination" (MMSE). Revista Portuguesa de Neurologia, 1, 9 .

Hawryluck, L., Gold, W. L., Robinson, S., Pogorski, S., Galea, S., \& Styra, R. (2004). SARS control and psychological effects of quarantine, Toronto, Canada. Emerging Infectious Diseases, 10(7), 1206-1212. https://doi.org/10.3201/eid1007.030703.

Hildon, Z., Montgomery, S. M., Blane, D., Wiggins, R. D., \& Netuveli, G. (2010). Examining resilience of quality of life in the face of health-related and psychosocial adversity at older ages: What is "right" about the way we age? The Gerontologist, 50(1), 36-47. https://doi.org/10.1093/geront/gnp067.

Huang, Y., \& Zhao, N. (2020). Generalized anxiety disorder, depressive symptoms and sleep quality during COVID-19 outbreak in China: A web-based cross-sectional survey. Psychiatry research, 112954. https://doi.org/10.1016/j.psychres.2020.112954.

IBM Corp. Released (2013). IBM SPSS statistics for windows, version 22.0. 8 Armonk, NY: IBM Corp.

Jeong, H., Yim, H. W., Song, Y. J., Ki, M., Min, J. A., Cho, J., \& Chae, J. H. (2016). Mental health status of people isolated due to Middle East respiratory syndrome. Epidemiology and health, 38, e2016048. https://doi.org/10.4178/epih.e201604828196409.

Liu, S., Yang, L., Zhang, C., Xiang, Y. T., Liu, Z., Hu, S., \& Zhang, B. (2020). Online mental health services in China during the COVID19 outbreak. The Lancet Psychiatry, 7(4), e17-e18. https://doi.org/ 10.1016/S2215-0366(20)30077-8.

Mak, I. W. C., Chu, C. M., Pan, P. C., Yiu, M. G. C., Ho, S. C., \& Chan, V. L. (2010). Risk factors for chronic post-traumatic stress disorder (PTSD) in SARS survivors. General Hospital Psychiatry, 32(6), 590-598. https://doi.org/10.1016/j.genhosppsych.2010.07.007.

Millan, M. J., Agid, Y., Brüne, M., Bullmore, E. T., Carter, C. S., Clayton, N. S., ... \& Dubois, B. (2012). Cognitive dysfunction in psychiatric disorders: Characteristics, causes and the quest for improved therapy. Nature Reviews Drug Discovery, 11(2), 141-168. https://doi.org/10.1038/nrd3628.

Neria, Y., Nandi, A., \& Galea, S. (2008). Post-traumatic stress disorder following disasters: A systematic review. Psychological Medicine, 38(4), 467-480. https://doi.org/10.1017/S0033291707001353.

Netuveli, G., \& Blane, D. (2008). Quality of life in older ages. British Medical Bulletin, 85(1), 113-126.

Nygren, B., Aléx, L., Jonsén, E., Gustafson, Y., Norberg, A., \& Lundman, B. (2005). Resilience, sense of coherence, purpose in life and self-transcendence in relation to perceived physical and mental health among the oldest old. Aging \& Mental Health, 9(4), 354-362.

O'luanaigh, C., O'connell, H., Chin, A. V., Hamilton, F., Coen, R., Walsh, C., et al. (2012). Loneliness and cognition in older people: The Dublin healthy ageing study. Aging \& Mental Health, 16(3), 347-352. https://doi.org/10.1080/13607863.2011.628977.

Pocinho, M., Farate, C., \& Dias, C. A. (2010). Validação Psicométrica da Escala UCLA-Loneliness para Idosos Portugueses. Interações, 8, 65-77.

Ribeiro, O., Paúl, C., Simões, M. R., \& Firmino, H. (2011). Portuguese version of the geriatric anxiety inventory: Transcultural adaptation and psychometric validation. Aging \& Mental Health, 15(6), 742748. https://doi.org/10.1080/13607863.2011.562177.

Ribeiro, O., Teixeira, L., Duarte, N., Azevedo, M. J., Araújo, L., Barbosa, S., \& Paúl, C. (2012). Versão portuguesa da escala breve de redes sociais de Lubben (LSNS-6). Revista Kairós: Gerontologia, 15(Especial11), 217-234.

Saltzman, L. Y., Hansel, T. C., \& Bordnick, P. S. (2020). Loneliness, isolation, and social support factors in post-COVID-19 mental health. Psychological trauma : theory, research, practice and policy, 12(S1), S55-S57. https://doi.org/10.1037/tra0000703. 
Simões, M. R., Freitas, S., Santana, I., Firmino, H., Martins, C., Nasreddine, Z., \& Vilar, M. (2008). Montreal Cognitive Assessment (MoCA): Versão final portuguesa. Serviço de Avaliação Psicológica, Faculdade de Psicologia e de Ciências da Educação da Universidade de Coimbra.

Sousa, L. B., Vilar, M., \& Simões, M. R. (2013). IAFAI, Inventário de Avaliação Funcional de Adultos e Idosos. Faculdade de Psicologia e de Ciências da Educação da Universidade de Coimbra.

Stead, A., \& Vinson, M. (2019). Cognitive assessment using face-to-face and videoconferencing methods. Nursing Older People, 31(5), 34 39. https://doi.org/10.7748/nop.2019.e1160.

The World Health Organization Quality of Life assessment (WHOQOL). (1995). Position paper from the World Health Organization. Social Science \& Medicine (1982), 41(10), 1403-1409. https://doi.org/10. 1016/0277-9536(95)00112-k.

Tilvis, R. S., Kähönen-Väre, M. H., Jolkkonen, J., Valvanne, J., Pitkala, K. H., \& Strandberg, T. E. (2004). Predictors of cognitive decline and mortality of aged people over a 10-year period. The Journals of Gerontology Series A: Biological Sciences and Medical Sciences, 59(3), M268-M274. https://doi.org/10.1093/gerona/59.3.M268.

Tull, M. T., Edmonds, K. A., Scamaldo, K. M., Richmond, J. R., Rose, J. P., \& Gratz, K. L. (2020). Psychological outcomes associated with stay-at-home orders and the perceived impact of COVID-19 on daily life. Psychiatry research, 289, 113098. Advance online publication. https://doi.org/10.1016/j.psychres.2020.113098.

Van Orden, K. A., Bower, E., Lutz, J., Silva, C., Gallegos, A. M., Podgorski, C. A., Santos, E. J., \& Conwell, Y. (2020). Strategies to promote social connections among older adults during 'Social Distancing' restrictions. The American journal of geriatric psychiatry : official journal of the American Association for Geriatric Psychiatry, https://doi.org/10.1016/j.jagp.2020.05.004. Advance online publication.

Vaz-Serra, A. (2000). Construção de uma escala para avaliar a vulnerabilidade ao Stress: a 23 GVS. Psiquiatria Clínica, 21(4), 279-308.
Venkatesh, A., \& Edirappuli, S. (2020). Social distancing in covid-19: What are the mental health implications? BMJ, 369(m1379). https:// doi.org/10.1136/bmj.m1379.

Vilar, M., Sousa, L. B., Freitas, S., Nogueira, J., Prieto, G., \& Simões, M. R. (2018). WHOQOL-OLD- 7. PsyLabAssessment, Faculdade de Psicologia e de Ciências da Educação da Universidade de Coimbra. Portugal.

Wang, H. X., Karp, A., Winbald, B., \& Fratiglioni, L. (2002). Late-life engagement in social and leisure activities is associated with a decreased risk of dementia. American Journal of Epidemiology, 155, 1081-1087. https://doi.org/10.1093/aje/155.12.1081.

Wechsler, D. (2008). Escala de Inteligência de Wechsler para Adultos Terceira edição (WAIS-III) [Wechsler adult intelligence scale-third edition]. Cegoc, Portugal.

White, R. G., \& Van Der Boor, C. (2020). Impact of the COVID-19 pandemic and initial period of lockdown on the mental health and well-being of adults in the UK. BJPsych open, 6(5), e90. https://doi. org/10.1192/bjo.2020.79.

Wilson, R. S., Krueger, K. R., Arnold, S. E., Schneider, J. A., Kelly, J. F., Barnes, L. L., Tang, Y., \& Bennett, D. A. (2007). Loneliness and risk of Alzheimer disease. Archives of General Psychiatry, 64(2), 234-240. https://doi.org/10.1001/archpsyc.64.2.234.

Xiao, H., Zhang, Y., Kong, D., Li, S., \& Yang, N. (2020). The effects of social support on sleep quality of medical staff treating patients with coronavirus disease 2019 (COVID-19) in January and February 2020 in China. Medical science monitor: international medical journal of experimental and clinical research, 26, e923549. https://doi.org/10.12659/MSM.923549.

Publisher's Note Springer Nature remains neutral with regard to jurisdictional claims in published maps and institutional affiliations. 